\title{
Mental health and physical health across the life span
}

\author{
Zum World Mental Health Day am 10. Oktober 2005
}

\author{
H. Kurt, Präsident Schweizerische Gesellschaft für Psychiatrie und Psychotherapie, SGPP \\ P. Haemmerle, Präsident Vereinigung psychiatrisch-psychotherapeutisch tätiger Ärzte und Ärztinnen FMPP \\ und Schweizerische Gesellschaft für Kinder- und Jugendpsychiatrie und -psychotherapie, SGKJPP
}

Psychische Gesundheit und Störungen derselben beginnen bereits intrauterin und beeinflussen danach unser Leben bis ins hohe Alter. Denken wir nur an die Wichtigkeit der frühkindlichen Entwicklung; danach an die Schulkinder, bei denen immer häufiger die Diagnose eines ADHD (Attention Deficit/Hyperactivity Disorder) gestellt wird; oder daran, dass Millionen von Kindern vor allem in Afrika an HIV/Aids erkrankt sind. Bei den Jugendlichen dann stehen Depressionen und Suchterkrankungen im Vordergrund, aber auch Essstörungen und Probleme rund um die Sexualität, wie z.B. frühe Schwangerschaften. Jugendliche und insbesondere junge Erwachsene haben hohe Suizidraten, die WHO schätzt, dass jährlich vier Millionen Jugendliche Suizidversuche begehen. Bei den Erwachsenen beeinträchtigen sodann Stress in der Arbeitswelt sowie konfliktreiche Familienverhältnisse das seelische Wohlergehen. Gewalt, Katastrophen, Flucht und Emigration belasten vor allem Frauen und Kinder, die $80 \%$ der rund 50 Millionen betroffenen Menschen ausmachen. Eine von fünf Frauen erleidet in ihrem Leben mindestens einmal sexuelle Gewalt. Männer wiederum - eigentlich eine Hochrisikogruppe - nehmen psychiatrisch-psychotherapeutische Hilfe kaum in Anspruch, gehen weniger zum Arzt, und wenn, dann klagen sie bei ihrem Arzt vorwiegend über körperliche Probleme. Bei älteren Menschen zeigen Untersuchungen, dass 15-25\% dieser Patienten an psychiatrischen Erkrankungen leiden.

Aus der gesamten Lebensspanne möchten wir zwei Altersgruppen hervorheben, nämlich Kleinkinder und alte Menschen. Beide werden primär von den Grundversorgern betreut; beide erfordern indessen eine spezielle Aufmerksamkeit hinsichtlich psychischer Symptome und eine entsprechende «ganzheitliche» Behandlung, welche die Einheit seelischer und körperlicher Faktoren hinsichtlich unserer Gesundheit berücksichtigt.
Es gibt klare, auch evidenzbasierte Studien, die zeigen, dass die postpartale Depression ein hohes Risiko für ein Misslingen der frühen Mutter-Kind-Beziehung darstellt und über lange Jahre die emotionale und kognitive Entwicklung des Kindes beeinträchtigen kann bis hin zur Herausbildung psychischer Erkrankungen. Wird die Depression jedoch frühzeitig diagnostiziert und entsprechend behandelt sowie die Beziehungsentwicklung der Mutter-Kind-Dyade gefördert, bleiben gravierende Spätfolgen meistens aus. Gesicherte Risikofaktoren für eine postpartale Depression sind psychiatrische Erkrankungen vor der Schwangerschaft, Armut, Gewalt in der Ehe sowie kulturelle Aspekte, wie etwa die Bevorzugung männlicher Nachkommen. Wenn wir diese Risikofaktoren kennen und erkennen, können wir präventiv aktiv werden und therapeutische Interventionen planen, auch wenn viele dieser Risikofaktoren eigentlich in den Bereich von Gesundheits- und Sozialpolitik gehören.

Wissenschaftliche Untersuchungen legen weiter nahe, dass Reifung und Entwicklung des menschlichen Gehirns eng zusammenhängen mit der Ausgestaltung der frühen Beziehungen, vor allem zur Mutter und zu den Eltern, später aber auch zu anderen Kindern und erwachsenen Bezugspersonen. Deshalb sind gelingende frühe Beziehungen ein wesentlicher Schutzfaktor, «Proviant» und Gewähr für eine gesunde Entwicklung des Kleinkindes. Babys zeigen noch nicht die typischen Symptome psychischer Störungen, sondern reagieren mit Schlafstörungen, Essschwierigkeiten, Unruhe, Bauchschmerzen und diffuser Ängstlichkeit. Wenn diese Symptome nicht oder zu spät erfasst und falsch eingeordnet werden, besteht die Gefahr, dass in späteren Jahren psychische Erkrankungen auftreten. Vorsorgeuntersuchungen, Früherkennung und Behandlung von Kleinkindern sollten deshalb nicht nur körperliche Aspekte von Gesundheit berücksichtigen, sondern ihr Augenmerk ebenso- 
sehr auf psychische Faktoren, soziale Bedingungen und erzieherische Aspekte in der ElternKind-Beziehung richten.

Aber selbst eine vermeintlich lebenslange gute psychische Gesundheit bietet keinen sicheren Schutz vor psychiatrischer Erkrankung im Seniorenalter, wie schwere Depressionen, Angsterkrankungen, Alzheimer-Demenzen oder andere Krankheitsbilder. Nach Schätzungen der WHO nehmen ältere Menschen gar eine Spitzenposition ein, was psychische Erkrankungen anbelangt. Bei den über 65jährigen Menschen leiden 2,5mal mehr Menschen an einer psychiatrischen Erkrankung, als dies bei den 45- bis 64jährigen der Fall ist. So machen die über 65jährigen in den USA 12\% der Bevölkerung aus, aber mehr als 20\% aller Suizide fallen in diese Altersgruppe. Über eine Million der älteren Amerikaner leiden an schweren hirnorganischen Beeinträchtigungen.

Auch hier gilt es, nicht nur Blutdruck, Cholesterin und andere somatische Parameter zu messen und $\mathrm{zu}$ behandeln, sondern eigentliche Stimmungs- und Gedächtnis-Check-ups sind genauso nötig. Die meist polypragmatische medikamentöse Behandlung bedarf einer speziellen Überwachung; die Behandlung soll möglichst lange zu Hause und in der Gemeinde ermöglicht werden; eine interdisziplinäre Zusammenarbeit mit Spitex-Pflege und anderen Betreuern ist unabdingbar; eine familienorientierte, wertschätzende Haltung gegenüber den Angehörigen - all dies spielt für das psychische und physische Wohlbefinden älterer Menschen eine grosse Rolle. Eine derart umfassende Behandlung liegt oft in den Händen des Hausarztes und der Hausärztin, die im besten Sinne des Wortes zu eigentlichen «Netzwerkern» werden. Ältere Menschen sollten gerade auch aus gesundheitlicher Sicht weiterhin einen sinnspendenden Platz in unserer Gesellschaft einnehmen können. Ihre Weisheit und ihre über lange Jahre gewachsene Erfahrung sind unschätzbare Werte in Familie und Gesellschaft, die es (wieder-)zu entdecken gilt!

Psychische Gesundheit und die Gefahr, sie zu verlieren, dauern ein ganzes Leben an und sind je nach Lebensphase verschieden ausgeprägt. Körperliche Symptome bilden oft nur «die Oberfläche» von Erkrankungen, denn unsere Gesundheit beruht unabdingbar auf einem engen Geflecht psychischer, körperlicher und sozialer Faktoren: Vergessen wir nie: "There is no health without mental health!" 